\title{
Neural correlates of focused attention in patients with mild Alzheimer's disease
}

\author{
Jennifer R. Bowes ${ }^{1 *}$, Patrick Stroman ${ }^{1,2,3}$, Angeles Garcia ${ }^{1,4}$ \\ ${ }^{1}$ Centre for Neuroscience Studies, Queen’s University, Kingston, Ontario, Canada \\ ${ }^{2}$ Department of Diagnostic Radiology, Queen’s University, Kingston, Ontario, Canada \\ ${ }^{3}$ Department of Physics, Queen's University, Kingston, Ontario, Canada \\ ${ }^{4}$ Department of Medicine, Queen's University, Kingston, Ontario, Canada \\ Email: ${ }^{\text {jbowes@cheo.on.ca }}$
}

Received 10 July 2012; revised 19 September 2012; accepted 29 September 2012

\begin{abstract}
Alzheimer's Disease (AD) is characterized by an early and significant memory impairment, and progresses to affect other cognitive domains. Impairments in Focused Attention (FA) have been observed in patients diagnosed with mild AD. A functional magnetic resonance imaging ( $f M R I)$ Stroop paradigm with verbal responses was used to investigate the neural correlates of FA in AD patients. Twenty-one patients diagnosed with mild AD performed a verbal StroopfMRI paradigm. Colour words were printed in an incongruent ink colour. Series 1 consisted of four blocks "Read the word" followed by four blocks "Say the colour of the ink"; Series 2 alternated between the two conditions. Functional data were analyzed using SPM5 to detect anatomical areas with significant signal intensity differences between the conditions. Withingroup analyses of the colour minus word contrast yielded significant activation in the following left hemisphere regions: precentral gyrus, inferior frontal gyrus, fusiform gyrus and supplementary motor area $(p<0.05$, uncorrected). Relative to cognitively normal older adults who underwent the same experimental task, Stroop performance was significantly worse in AD patients. The $f M R I$ task yielded similar activated brain regions between the two groups. The use of verbal responses in this novel $f$ MRI Stroop task avoids the confusion and memorizing of button locations seen with the manual response modality, allowing the neural correlates of FA to be investigated in AD patients.
\end{abstract}

Keywords: fMRI; Focused Attention; Stroop; Alzheimer's Disease

\section{INTRODUCTION}

Alzheimer's Disease (AD) is a neurodegenerative disease

"Corresponding author. with significant memory impairment as the principal clinical feature. Clinicians use the term $\mathrm{AD}$ to refer to a clinical entity that typically presents with a progressive amnestic disorder with subsequent appearance of other cognitive, behavioural, and neuropsychological changes that impair social function and activities of daily living [1]. Among the cognitive changes that occur, deficits in attention, which restricts the sensory input load that enters our processing system, have been recognized to occur in the early stages of $\mathrm{AD}$ [2]. Focused Attention (FA), one of the subcomponents of attention, modulates incoming stimuli to enhance the relevant information and inhibit the irrelevant information.

The Stroop task [3] is a well-known experimental paradigm considered the gold standard assessment of FA. It is often used clinically as part of a neuropsychological test battery to assess the cognitive functioning in $\mathrm{AD}$ patients. The Stroop task measures the ability to inhibit irrelevant information by presenting colour names printed in a non-matching (incongruent) ink colour [4]. When asked to name the colour of the ink, an efficient FA system suppresses the irrelevant (the written word) resulting in naming of the relevant (ink colour) dimension [5]. Young adults perform significantly better on the Stroop task than cognitively normal older adults $[5,6]$, who in turn perform significantly better than AD patients [5,7-9]. Impairments in FA reveal themselves in the Stroop task by an increase in response latency and/or an increase in errors when naming the colours of the ink $[5,10]$. Poor performance on measures of FA in early AD may reflect a greater pathology in frontal lobes and associated areas than would be anticipated given the predominant pathology of AD in the medial temporal lobes [11].

Functional Magnetic Resonance Imaging ( $\mathrm{fMRI}$ ) is a non-invasive neuroimaging technique that provides relatively high spatial resolution in order to identify which regions of the brain activity correlate with a given cognitive task [12]. Over the past decade, the Stroop task has 
been combined with $f$ MRI methodology to allow for the study of the neural substrates underlying FA. In a metaanalysis of functional neuroimaging studies of the Stroop task in normal subjects, Laird et al. [13] found activation in the bilateral anterior cingulate cortex, left inferior and middle frontal, and left inferior parietal regions of controls. While $f$ MRI provides a powerful method to study how the brain functions during the Stroop task in controls, it can also be used to see how brain activation may be altered in different patient populations. Neuroimaging of patients diagnosed with $\mathrm{AD}$ while doing a cognitive task may prove challenging and requires an experimental paradigm that the patients can comprehend in order to perform the task. Most of the studies of Stroop-fMRI in cognitively normal older adults have been done using a manual (button box) modality [14-16]. However, by self-report and performance, older adults had more difficulty using the manual modality than younger adults [16]. Given that the manual modality adds additional cognitive demands by requiring participants to remember which button corresponds to which colour stimuli, AD patients may find the manual modality too confusing to operate. Use of the verbal modality of the Stroop task, which requires participants to speak the words aloud, is more similar to the paper version, and may be a more appropriate modality for $\mathrm{AD}$ patients.

The current study employed a novel verbal modality Stroop-fMRI paradigm, used previously in cognitively normal older adults [17] to assess the neural substrates underlying Stroop task performance in mild AD patients.

\section{METHODS}

\subsection{Subject Population}

Twenty-one patients (12 women, 9 men; mean age $73 \pm 9$ ) diagnosed with mild $\mathrm{AD}$ were recruited from an outpatient memory disorders clinic and underwent the experimental task. All participants were native or highly proficient English-speaking volunteers between 51 and 84 years of age. All participants gave written informed consent prior to undergoing any study procedures and could safely undergo a MRI, with no colour blindness, neurological or other medical conditions that could interfere with the experimental task. Demographic and cognitive test scores for all $21 \mathrm{AD}$ patients are reported. Due to a large number of errors during the fMRI task, six AD patients were excluded from the subsequent $f$ MRI task analysis. Therefore, the $f$ MRI task results herein describe the 15 patients (10 women, 5 men), with a mean age of $73 \pm 9$, who successfully completed the experimental task. Twentyone cognitively normal older adults who performed the same experimental task, and whose results were described previously [17] were used as comparative data for the AD patients. The study was approved by the University Research Ethics Board.

\subsection{Cognitive Assessment}

Patients diagnosed with $\mathrm{AD}$, with Montreal Cognitive Assessment (MoCA) [18] scores between 15 and 26 at the time of the $f \mathrm{MRI}$, and who were capable of comprehending and performing the Stroop test, were approached for study participation. The diagnosis of $\mathrm{AD}$ was made according to the Diagnostic and Statistical Manual for Mental Disorders, $4^{\text {th }}$ edition (DSM-IV) [19] and National Institute of Neurologic and Communicative Disorders and Stroke/Alzheimer's Disease and Related Disorders Association (NINCDS-ADRDA) [20] criteria for clinically probable $\mathrm{AD}$. The diagnosis was made or confirmed by a geriatrician (AG) in the memory disorders clinic. Participants enrolled in the study underwent a series of neuropsychological tests to assess their general cognitive function, which included the Mini-Mental State Examination (MMSE) [21], MoCA, the Mattis Dementia Rating Scale (DRS) [22], and the Stroop test.

\section{3. fMRI Experimental Task}

The fMRI experiment was carried out at the Queen's University Research MRI Facility. The Stroop test used was an adaptation from the word-colour incongruent task of the paper version of the Stroop test. An MR-compatible microphone with noise cancellation was placed in front of the participant's mouth to record the verbal responses to the presented colour word stimuli. Participants were given headphones to wear to reduce the noise of the MR scanner and to allow for communication with the participant while they were in the scanner. Participants were instructed to speak aloud, but quietly, and without excessive enunciation, in an attempt to minimize head motion while speaking. Verbal reminders to keep still were made at short rest intervals between imaging series, and padding between the outside of the head-phones and the sides of the head coil reduced the opportunity for head movement. The experimental protocol was carried out with four different block design presentations, referred to as "series" as displayed in Figure 1. Condition blocks were 28 seconds and consisted of 16 word stimuli, each displayed for 1.75 seconds. Condition blocks were separated by 14 -second rest periods. The total experimental run took 20 minutes. Participants were instructed to either "Read the word" (herein referred to as "Word" condition) or "Say the Colour of the Ink" (herein referred to as the "Colour" condition). In Series 0, 4 blocks of colour word stimuli were presented in black ink and participants were asked to "Read the word". In Series 1, 8 blocks of colour word stimuli were presented in an incongruent ink colour. In the first 4 blocks, partici- 


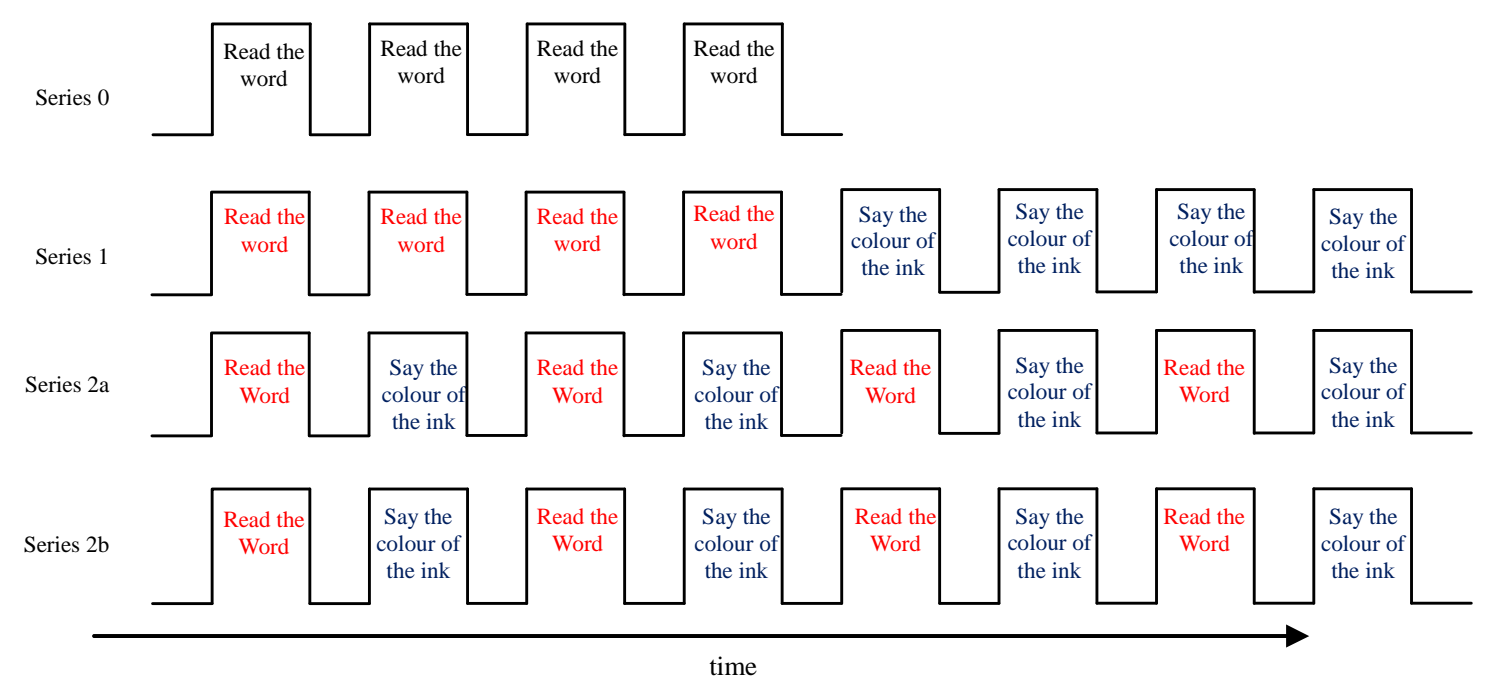

Figure 1. Stroop-fMRI experimental paradigm. In Series 0, 4 blocks of colour word stimuli were presented in black ink and participants were asked to read the word. The first rest period displayed the instruction of "Read the word"; the remaining rest periods displayed a fixation cross. Series $1,2 \mathrm{a}$, and $2 \mathrm{~b}$ had 8 blocks of colour word stimuli presented in an incongruent ink colour, with the rest period displaying the instructions to the subsequent condition block. In Series 1, participants were asked to "Read the word" for the first 4 blocks, and to "Say the colour of the ink" for the last 4 blocks. Series 2a and 2b were identical and blocks alternated between the "Read the word" and "Say the colour of the ink" conditions. Each condition block was 28 seconds, and rest periods between conditions blocks were 14 seconds. Series 0 was 182 seconds long and Series 1, 2a and 2b were each 350 seconds long.

pants were asked to "Read the word" and in the last 4 blocks, participants were asked to "Say the colour of the ink". Series 2a and Series $2 \mathrm{~b}$ alternated between the two conditions, each with 8 blocks of colour word stimuli presented in an incongruent ink colour. For Series 1, 2a and $2 \mathrm{~b}$, each rest period displayed the instructions for the subsequent condition block. This Stroop-fMRI experiment has been previously described in detail [17].

\subsection{Image Acquisition}

All images were acquired with a 3 Tesla Siemens Magnetom Trio MRI system (Siemens Medical Systems, Erlangen, Germany) using a 12-channel receive-only head coil. Motion correction software was applied during scanning, and also in the subsequent data analysis. High-resolution structural images were acquired with a T1weighted 3D, MP-RAGE sequence (ascending sequence in the axial plane with 176 slices, $\mathrm{TR}=1760 \mathrm{msec}$, TE $=$ $2.2 \mathrm{~m} \cdot \mathrm{sec}$, flip angle $=9^{\circ}, \mathrm{FoV}=256 \mathrm{~mm}$, voxel size $=1$ $\mathrm{mm}^{3}$ ). Functional data were acquired with Blood Oxygenation-Level Dependent (BOLD) sensitive ( $\mathrm{T}_{2}^{*}$-weighted) echo planar imaging. Thirty-two axial slices were acquired, oriented parallel to the anterior commissure-posterior commissure (AC-PC) line, providing whole-brain coverage $\left(\mathrm{TR}=2000 \mathrm{~m} \cdot \mathrm{sec}, \mathrm{TE}=30 \mathrm{~m} \cdot \mathrm{sec}\right.$, flip angle $=78^{\circ}$, $\mathrm{FoV}=211 \mathrm{~mm}, 3.3 \mathrm{~mm}$ cubic voxels).

\subsection{Data Analysis}

Statistical analysis of demographics, cognitive assess- ment and performance accuracy was performed using SPSS, Version 17.0 (SPSS Inc., Chicago, IL). Participant's verbal responses to colour word stimuli were recorded for analysis of performance accuracy on the Stroop-fMRI task. Condition blocks with less than 75\% accuracy were excluded from $f$ MRI analysis. Errors were classified as incorrect, missed or corrected responses.

Spatial pre-processing and statistical analysis of the imaging data was carried out using SPM5 (Wellcome Department of Cognitive Neurology, University College London, England). The spatial pre-processing performed on the functional data was previously described in detail [17]. Statistical analysis was performed using the univariate approach of the general linear model [23] whereby the box-car reference function is convolved with the hemodynamic response function to account for the delay in the BOLD signal. For each participant, a design matrix was created with the rest periods, correct "Word" blocks and correct "Colour" blocks applied as regressors, in addition to the six realignment parameters. Error blocks were represented as separate regressors in the design matrix, though not used in any contrasts. Voxel-wise statistical analysis was then carried out to determine which voxels were activated by the different experimental conditions. Fixed-effects analysis was applied to each participant resulting in Statistical Parametric Maps (SPMs) for every contrast of interest, using a significance threshold of $p=$ 0.001 , uncorrected for multiple comparisons. Individual SPMs from each participant were then pooled together and entered into a random-effects analysis. A one-sample 
$t$-test was applied to investigate the within group activation level with a significance threshold of $p=0.05$, uncorrected for multiple comparisons. To control for false positives, a spatial extent cluster threshold of 3 voxels was applied. The coordinates of voxels that survived the statistical threshold were entered into MRICro software [24], which uses an Automated Anatomical Labeling (AAL) template [25] to report the localization of the activation. Significant voxels reported in SPM5 are reported in MNI space, and the AAL template was created based on the anatomical parcellation of the spatially normalized single-subject, segmented high resolution T1 volume provided by MNI. Realignment parameters calculated from motion-corrected and non motion-corrected functional data were used to estimate participant motion during the experimental task.

\section{RESULTS}

\subsection{Behavioural Results}

Demographic descriptives and mean neuropsychological test battery scores are listed in Table 1. In the paper version of the Stroop task administered as part of the test battery, all participants were significantly better reading the word than naming the colour of the ink $(p<0.001)$.

Both non-motion corrected and motion corrected data were inspected to determine the potential influence of participant motion on the results. The average participant movement was estimated based on the rotation about and the translation along each of the 3 coordinate axes $(x, y$, and $z)$. There was a mean $( \pm S D)$ translation of $5.7 \pm 4.39$ $\mathrm{mm}$ and a rotation of $6.13 \pm 2.74$ degrees for non-motioncorrected acquired images. For the motion corrected acquired images, the mean $( \pm \mathrm{SD})$ translation was $3.23 \pm$ $2.95 \mathrm{~mm}$ and a rotation of $3.6 \pm 2.21$ degrees. Due to the smaller motion parameters in the motion corrected images, these images were used in the analysis of the func-

Table 1. Demographic descriptives and mean neuropsychological test battery scores.

\begin{tabular}{lc}
\hline & $\begin{array}{c}\text { Alzheimer's disease patients } \\
N=21 \text { Mean (SD) }\end{array}$ \\
\hline Gender (M:F) & $9: 12$ \\
Age (years) & $73(9)$ \\
Education (years) & $16(5)$ \\
MMSE (/30) & $26.48(2.71)$ \\
MoCA (/30) & $21.38(3.06)$ \\
DRS ${ }^{*}$ (/144) & \\
Total Score & $129.18(5.99)$ \\
Stroop (/112) & \\
$\quad$ Word Score & $108.43(16.14)$ \\
$\quad$ Colour Score & $64.29(21.35)$ \\
\hline
\end{tabular}

$\mathrm{M}=$ Male $\mathrm{F}=$ Female MMSE $=$ Mini-Mental State Examination; MoCA = Montreal Cognitive Assessment; DRS = Dementia Rating Scale; ${ }^{*} \mathrm{~N}=17$. tional data. The average motion is comparable to the dimensions of one voxel in the data.

Performance accuracy was assessed by looking at the number of errors made during the Stroop-fMRI task. The mean $( \pm S D)$ total number of errors for the mild AD patients across all series was $50.33 \pm 30.42$. The mild AD patients made significantly more errors in the Stroop$f$ MRI task than cognitively normal older adults (mean total number of errors $8.00 \pm 6.83$ ) who performed the same task $(p<0.001)$ [17]. The mean $( \pm \mathrm{SD})$ number of errors in the incongruent colour-word trials of Series 1, Series $2 \mathrm{a}$ and Series $2 \mathrm{~b}$ were $11.47 \pm 10.86,25.20 \pm$ 18.19 and $13.67 \pm 14.02$, respectively. Of the three incongruent colour-word series, Series 1 showed the fewest errors and Series 2a showed the most errors. Series $2 b$ showed an improvement from Series 2a but had more errors than Series 1. The total number of errors by series comparing the $\mathrm{AD}$ patients to the cognitively normal older adults is displayed in Figure 2. There was no differrence in the number of errors across the incongruent "Word" and "Colour" conditions $(p=0.994)$ with a mean $( \pm$ SD) of $25.13 \pm 24.14$ and $25.20 \pm 19.88$, respectively. When the number of errors by condition was stratified by Series, as shown in Figure 2, only Series 1 showed significantly more errors in the "Colour" condition than in the "Word" condition $(p=0.004)$. The mean $( \pm S D)$ number of incorrect, corrected, and missed responses were $10.24 \pm 12.39,0.87 \pm 0.94,5.67 \pm 7.00$, respectively.

\subsection{Imaging Results}

Across the three incongruent series (Series 1, 2a and 2b), brain activation was significantly higher (i.e. greater number of active voxels) in the "Colour" condition than in the "Word" condition. The main contrast of interest was to identify which brain areas were more activated in the incongruent "Colour" condition than in the "Word" condition. The within-group analysis of the main con-

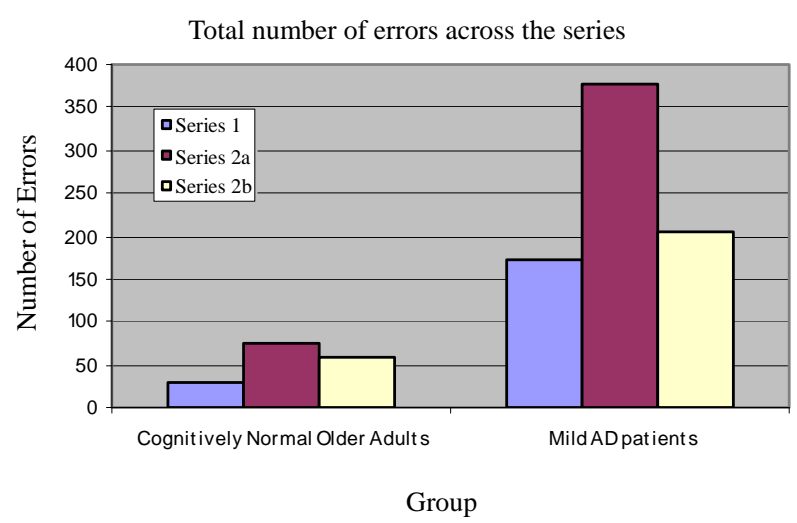

Figure 2. Total number of errors across the incongruent colourword series for cognitively normal older adults and mild AD patients. The distribution of errors across the three series was similar for both groups. 
trast of interest yielded greater activation in the left precentral gyrus, left pars opercularis of the inferior frontal gyrus (IFG), left pars triangularis of the IFG, pars orbital of the IFG, left fusiform gyrus, and left supplementary motor area (SMA), as listed in Table 2. Those brain areas found to be significantly more active in the "Colour" condition are displayed on a SPM and a template brain in Figure 3 ( $p=0.05$, uncorrected for multiple comparisons). In Series 1, where participants made significantly more errors in the "Colour" condition than the "Word" condition, the main contrast resulted in significant activation in the left precentral gyrus, left middle and superior frontal gyrus, bilateral fusiform gyrus, right insula, left hippocampus and parahippocamal, left superior parietal, bilateral inferior temporal gyrus, bilateral precuneus, left SMA, and left pars orbital and pars triangularis of the IFG. The largest activation cluster for the main contrast in Series 1 was 301 voxels. At a $p$ value of 0.001 significance threshold, only the left precentral gyrus survived statistical significance for the within group main contrast in Series 1. Combining the alternating incongruent colourword series, Series $2 \mathrm{a}$ and $2 \mathrm{~b}$, the main contrast yielded significant activation in the left pars orbital and pars triangularis of the IFG, left middle frontal gyrus, left precentral and left SMA. The largest activation cluster for the main contrast in the combined Series $2 \mathrm{a}$ and $2 \mathrm{~b}$ was 29 voxels.

The patterns of brain activation for the main contrast of interest in the mild $\mathrm{AD}$ patients were compared to those of the cognitively normal older adults previously described [17]. The brain regions that were significantly more activated in the cognitively normal older adults group were the right middle frontal gyrus, left middle occipital gyrus, right pars opercularis of the IFG, bilateral lingual and pars triangularis of the IFG. The brain regions that were significantly more activated in the mild $\mathrm{AD}$ patients were the left precentral, left supramarginal gyrus, left postcentral gyrus and the right middle frontal gyrus.

In Series 0, colour word stimuli were presented in black ink and condition blocks alternated with rest blocks whereby a fixation cross was displayed. A within group analysis of the "Word" minus fixation cross contrast yielded significant activity in brain regions including the left superior parietal, right precentral gyrus, right middle and superior frontal gyrus, bilateral SMA, left lingual and left postcentral gyrus.

\section{DISCUSSION}

This Stroop-fMRI study used a novel experimental paradigm to investigate the neural correlates of FA in $\mathrm{AD}$ patients. This paradigm, which had been previously employed in cognitively normal older adults, can be administered and performed by patients with cognitive impairments, as demonstrated by the $\mathrm{AD}$ patients in this study.

\subsection{Behavioural Results}

As designed, results from the neuropsychological test battery indicate relatively good cognitive functioning among the participants, despite a diagnosis of AD. The mild $\mathrm{AD}$ patients demonstrated the Stroop effect on the paper version of the Stroop task administered as part of the battery of neuropsychological tests. Patients with mild AD made more errors when asked to name the colour of the ink compared to naming the written word. This is thought to reflect the increase in cognitive interference in overcoming the more automatic response of reading the word to name the ink colour. As expected, given the effect of AD on cognitive functioning, and as previously shown [5,7-9], mild AD patients performed significantly worse when naming the colour of the ink on the paper version the Stroop task than cognitively normal older adults $(p<0.001)$.

When errors across all the incongruent colour word trials in the Stroop-fMRI experimental task were analyzed together, there was no significant difference in errors between the "Colour" and "Word" conditions ( $p=$ 0.994). However, once the errors were stratified by series (Series 1, 2a and 2b), only Series 1 , which consisted of 4

Table 2. Within-group analysis of the mild Alzheimer's disease (AD) patients yielding brain areas more activated in the "Colour" than in the "Read" condition.

\begin{tabular}{|c|c|c|c|c|c|c|}
\hline \multirow{2}{*}{ Region } & \multirow{2}{*}{ Side } & \multicolumn{3}{|c|}{ MNI coordinates in Talairach space $(\mathrm{mm})$} & \multirow{2}{*}{$\mathbf{T}$} & \multirow{2}{*}{ Z-score } \\
\hline & & $x$ & $y$ & $\mathbf{z}$ & & \\
\hline Precentral gyrus & $\mathrm{L}$ & -48 & 6 & 48 & 3.63 & 2.99 \\
\hline Inferior frontal gyrus, pars opercularis & $\mathrm{L}$ & -51 & 24 & 33 & 2.52 & 2.25 \\
\hline Inferior frontal gyrus, pars triangularis & $\mathrm{L}$ & -39 & 18 & 27 & 2.09 & 1.92 \\
\hline Inferior frontal gyrus, pars orbital & $\mathrm{L}$ & -42 & 24 & -15 & 2.62 & 2.32 \\
\hline Fusiform gyrus & $\mathrm{L}$ & -27 & -60 & -15 & 2.60 & 2.31 \\
\hline \multirow{2}{*}{ Supplementary motor area } & \multirow{2}{*}{$\mathrm{L}$} & -6 & 12 & 48 & 2.28 & 2.07 \\
\hline & & 0 & 3 & 69 & 1.94 & 1.80 \\
\hline
\end{tabular}



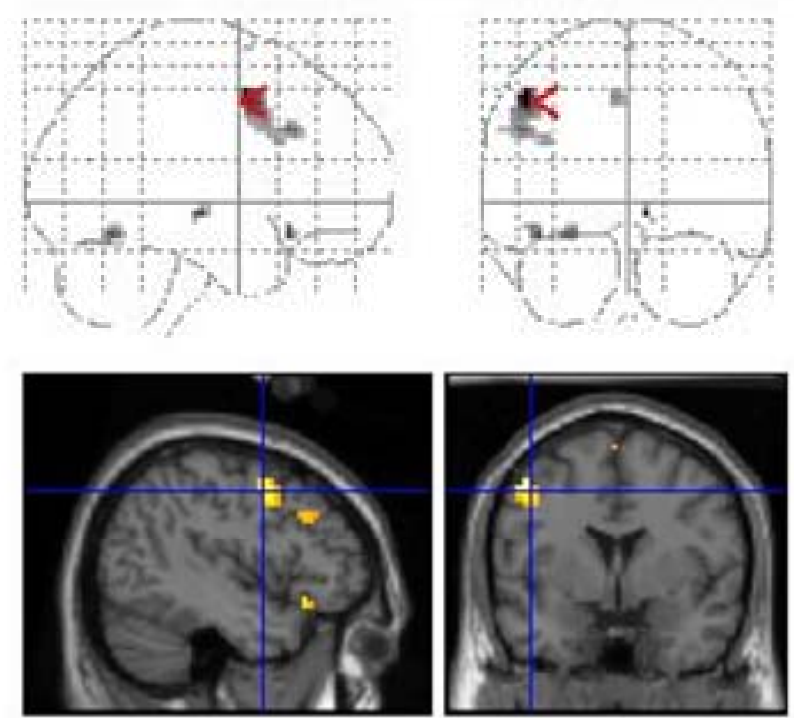

Figure 3. Overlay of brain activation of the within-group analysis for the "Say the colour of the ink" minus "Read the word" contrast on a SPM glass brain (top) and on an anatomical template (bottom).

consecutive blocks of the "Word" condition followed by 4 consecutive blocks of the "Colour" condition did AD patients show significantly more errors in the "Colour" condition than the "Word" condition. This was the case for both cognitively normal older adults and AD patients, and is likely to reflect the study paradigm. Series 1 is the series that is most similar to the paper version of the Stroop task, and may explain why only this series yielded the Stroop effect (e.g. significantly more errors when naming the ink colour than reading the word). The distribution of errors across the three series was also similar between the $\mathrm{AD}$ patients and cognitively normal older adults, with the alternating "Word" and "Colour" conditions Series $2 \mathrm{a}$ and $2 \mathrm{~b}$ showing the highest number of errors. Despite these similarities in task performance accuracy, AD patients had lower Stroop task performance than the cognitively normal older adults, as assessed by the number of error responses. This was true for both the colour naming part of the paper Stroop test and the Stroop-fMRI task, and may be a consequence of impairments of FA.

Errors were classified as incorrect, missed or corrected. Incorrect responses were the most common type of error by AD patients followed by missed responses. Fisher et al. [8] measured correct responses within a 45-second interval during Stroop performance in healthy older adults and $\mathrm{AD}$ patients and found that the $\mathrm{AD}$ patients made fewer correct responses. Reaction time was not measured in this study as colour word stimuli were presented every 1.75 seconds regardless of whether a verbal response was made. Therefore, missed responses may reflect a slower reaction time by $\mathrm{AD}$ patients when pre- sented with conflicting colour information. Previous studies of FA in AD patients have revealed increased reaction time compared to normal controls $[5,26]$.

The introduction of Series 2a, with alternating condition blocks, resulted in the highest number of errors for $\mathrm{AD}$ patients. In Series $2 \mathrm{~b}$, the second series of alternating condition blocks, participants made fewer errors than 2a, but still more than the non-alternating Series 1 . Due to the continuous switching between "Word" and "Colour" condition blocks in Series 2a and 2b, the high rate of errors likely reflects the additional cognitive demands required to maintain the proper task instructions. This would be especially true for those participants with memory impairments, such as the AD patients, who would have increased difficulty remembering which instructions were given at the start of each block. In their study of attentional processing in AD patients, Fernandez-Duque \& Black [27] found that AD patients were impaired in their ability to maintain an alternating mental set in the presence of distraction. Moreover, AD patients had difficulty keeping in mind the rule for target selection, especially when the rule changed throughout the experiment. This may hold true for the AD patients who participated in the current study, and may account for their high numbers of errors.

\subsection{Imaging Results}

The main contrast of interest aimed to determine which brain regions were activated in the "Colour" condition that were not activated during the "Word" condition. For $\mathrm{AD}$ patients, this contrast showed activation in the precentral gyrus, IFG, fusiform gyrus and SMA when asked to name the colour of the ink. The IFG was found to be activated in several previous Stroop-fMRI studies [14,15]. In addition, Milham et al. [15] found the SMA showed increased activity specific to the presence of conflicting colour information. Incongruent colour word trials have demonstrated activation in the bilateral inferior frontal sulcus, bilateral intraparietal sulcus, the posterior superior frontal gyrus and the medial wall of the superior frontal gyrus [14]. Furthermore, the center of activation in the IFG was located in the pars triangularis, and reaching into the pars opercularis [14]. These brain regions were similarly activated in the current study, and may reflect the predominantly incongruent colour word trials of the Stroop-fMRI paradigm. The activation of the fusiform gyrus has been shown in previous Stroop-fMRI studies, and likely reflects its role in processing visual information, namely of words and of colour perception [14,28-30].

Similar brain regions found activated in this study's AD patient population and in previous studies of cognitively normal older adults likely reflects a network of brain regions activated in order to overcome the cogni- 
tive interference presented by the Stroop task. Milham et al. [15] suggests that as the need for attentional control increased across conditions, older adults activated a network of structures in order to perform the task. Compared to the cognitively normal older adults who performed the same verbal modality Stroop-fMRI paradigm, $\mathrm{AD}$ patients appeared to recruit additional brain regions, primarily left hemisphere frontal regions, in the face of Stroop interference. Although the bulk of evidence points to the left hemisphere for the resolution of the interference [31], AD patients appeared to rely more exclusively on it than cognitively normal older adults.

Common brain regions in the main contrast between the current study and the previous Stroop-fMRI studies were identified despite obvious differences in experimental paradigms. For example, a verbal response modality was used in the current study. Although there appears to be a network of brain regions that are activated in order to facilitate the Stroop task regardless of modality, variations in the regions activated could be attributed to methodological differences. However, if the modality used in Stroop-fMRI paradigms does not impact greatly on the network of brain regions activated, the use of the verbal modality should be considered, especially when studying populations that may have difficulties using the manual button box. While controlling for and using motion correction tools to overcome the motion artifact from the verbal responses, the current Stroop-fMRI experimental paradigm has been successfully performed by both cognitively normal older adults, and by patients with mild AD.

\section{CONCLUSION}

In the current study, mild AD patients successfully performed a Stroop-fMRI experimental task using a verbal response modality in order to investigate the neural correlates of FA in this patient population. Although $\mathrm{AD}$ patients activated some of the same brain regions as cognitively normal older adults, a higher number of errors suggest impairments in FA in this patient population. Despite mild AD patients with relatively high cognitive functioning, deficits in FA were observed in this study.

\section{ACKNOWLEDGEMENTS}

The study was supported by a Queen's University CTAQ Endowment Fund grant.

\section{REFERENCES}

[1] Cummings, J.L. (2004) Alzheimer's disease. New England Journal of Medicine, 351, 56-67. doi:10.1056/NEJMra040223

[2] Baddeley, A.D., Baddeley, H.A., Bucks, R.S. and Wil- cock, G.K. (2001) Attentional control in Alzheimer's disease. Brain, 124, 1492-1508.

doi:10.1093/brain/124.8.1492

[3] Stroop, O.R. (1935). Studies of interference in serial verbal reaction. Journal of Experimental Psychology, 18, 643662. doi:10.1037/h0054651

[4] Perry, R.J. and Hodges, J.R. (1999) Attention and executive deficits in Alzheimer's disease: A critical review. Brain, 122, 383-404. doi:10.1093/brain/122.3.383

[5] Spieler, D., H., Balota, D.A. and Faust, M.E. (1996) Stroop performance in healthy younger and older adults and in individuals with dementia of the Alzheimer's type. Journal of Experimental Psychology: Human Perception and Performance, 22, 461-479. doi:10.1037/0096-1523.22.2.461

[6] West, R. and Baylis, G.C. (1998) Effects of increased response dominance and contextual disintegration on the stroop interference effect in older adults. Psychology and Aging, 13, 206-217. doi:10.1037/0882-7974.13.2.206

[7] Koss, E., Ober, B.A., Delis, D.C. and Friedland, R.P. (1984) The stroop color-word test: Indicator of dementia severity. International Journal of Neuroscience, 24, 5361. doi:10.3109/00207458409079534

[8] Fisher, L.M., Freed, D.M. and Corkin, S. (1990) Stroop color-word test performance in patients with Alzheimer's disease. Journal of Clinical Experimental Neuropsychology, 12, 745-758. doi:10.1080/01688639008401016

[9] Bondi, M.W., Serody, A.B., Chan, A.S., EbersonShumate, S.C., Delis, D.C., et al. (2002) Cognitive and neuropathologic correlates of stroop color-word test performance in Alzheimer's disease. Neuropsychology, 16, 335343. doi:10.1037/0894-4105.16.3.335

[10] Kane, M.J. and Engle, R.W. (2003) Working-memory capacity and the control of attention: The contribution of goal neglect, response competition, and task set to stroop interferences. Journal of Experimental Psychology General, 132, 47-70. doi:10.1037/0096-3445.132.1.47

[11] McGuinness, B., Barrett, S.L., Craig, D., Lawson, J. and Passmore, A.P. (2010) Attentional deficits in Alzheimer's disease and vascular dementia. Journal of Neurology, Neurosurgery, and Psychiatry, 81, 157-159. doi:10.1136/jnnp.2008.164483

[12] Samanez-Larkin, G.R. and D’Esposito, M. (2008) Group comparisons: Imaging the aging brain. Social Cognitive and Affective Neuroscience, 3, 290-297. doi:10.1093/scan/nsn029

[13] Laird, A.R., McMillan, K.M., Lancaster, J.L., Kochunov, P., Turkeltaub, P.E., Pardo, J.V., et al. (2005) A Comparison of label-based review and ale meta-analysis in the Stroop task. Human Brain Mapping, 25, 6-21. doi:10.1002/hbm.20129

[14] Zysset, S., Schroeter, M.L., Neumann, J. and von Cramon, D.Y. (2007) Stroop interference, hemodynamic response and aging: An event-related fMRI study. Neurobiology of Aging, 28, 937-946. doi:10.1016/j.neurobiolaging.2006.05.008

[15] Milham, M.P., Erikson, K.I. Banich, M.T., Kramer, A.F., Webb, A., Wszalek, T. and Cohen, N.J. (2002) Atten- 
tional control in the aging brain: Insights from an fMRI study of the Stroop task. Brain and Cognition, 49, 277296. doi:10.1006/brcg.2001.1501

[16] Langenecker, S.A., Nielson, K.A. and Rao, S.M. (2004) fMRI of healthy older adults during Stroop interference. Neuro Image, 21, 192-200. doi:10.1016/j.neuroimage.2003.08.027

[17] Bowes, J., Stroman, P., and Garcia, A. (2011) Neural correlates of focused attention in cognitively normal older adults. World Journal of Neuroscience, 1, 19-27. doi:10.4236/wjns.2011.12003

[18] Nasreddine, Z.S., Phillips, N.A., Bédirian, V., Charbonneau, S., Whitehead, V., Collin, I., Cummings, J.L. and Chertkow, H. (2005) The montreal cognitive assessment, MoCA: A brief screening tool for mild cognitive impairment. Journal of the American Geriatrics Society, 53, 695-699. doi:10.1111/j.1532-5415.2005.53221.x

[19] American Psychiatric Association (1994) Diagnostic and statistical manual of mental disorders. 4th Edition, American Psychiatric Association, Washington DC.

[20] McKhann, G., Drachmna, D., Folstein, M., Katzman, R., Price, D. and Stadlan, E. (1984) Clinical diagnosis of Alzheimer's disease: Report of the NINCDS-ADRDA work group under the auspices of department of health and human services task force on Alzheimer's disease. $\mathrm{Neu}$ rology, 34, 939-944. doi:10.1212/WNL.34.7.939

[21] Folstein, M., Folstein, S. and McHugh, P.R. (1975) Minimental state: A practical method for grading the cognitive state of patients for the clinician. Journal of Psychiatry Research, 12, 189-198. doi:10.1016/0022-3956(75)90026-6

[22] Mattis, S. (1988). Dementia Rating Scale. Professional Manual. Psychological Assessment Resources, Odessa.

[23] Friston, K.J., Holmes, A.P., Worsley, K.J., Poline, J.P., Frith, C.D. and Frackowiak, R.S.J. (1994) Statistical parametric maps in functional imaging-A general linear approach. Human Brain Mapping, 2, 189-210. doi:10.1002/hbm.460020402
[24] Rorden, C. and Brett, M. (2000) Stereotaxic display of brain lesions. Behavioural Neurology, 12, 191-200.

[25] Tzourio-Mazoyer, N., Landeau, B., Papathanassiou, D., Crivello, F., Etard, O., Delcroix, N., Mazoyer, B. and Joliot, M. (2002) Automated anatomical labeling of activations in SPM using a macroscopic anatomical parcellation of the MNI MRI single-subject brain. NeuroImage, 15, 273-289. doi:10.1006/nimg.2001.0978

[26] Levinoff, E.J., Saumier, D. and Chertkow, H. (2005) Focused attention deficits in patients with Alzheimer's disease and mild cognitive impairment. Brain and Cognition, 57, 127-130. doi:10.1016/j.bandc.2004.08.058

[27] Fernandez-Duque, D. And Black, S.E. (2008) Selective attention in early dementia of Alzheimer type. Brain and Cognition, 66, 221-231. doi:10.1016/j.bandc.2007.08.003

[28] Banich, M.T., Milham, M.P., Atchley, R., Cohen, N.J., Webb, A., Wszalek, T., et al. (2000). fMRI studies of Stroop tasks reveal unique roles of anterior and posterior brain systems in attentional selection. Journal of Cognitive Neuroscience, 12, 988-1000. doi:10.1162/08989290051137521

[29] Banich, M.T., Milham, M.P., Jacobson, B.L., Webb, A., Wszalek, T., Cohen, N.J., et al. (2001). Attentional selection and the processing of task-irrelevant information: Insights from fMRI examinations of the Stroop task. In: Casanova, C. and Pt Ptito, M., Eds., Vision: From Neurons to Cognition, Elsevier Science, Amsterdam, 459470. doi:10.1016/S0079-6123(01)34030-X

[30] Taylor, S.F., Korblum, S., Lauber, E.J., Minoshima, S. and Koeppe, R.A. (1997) Isolation of specific interference processing in the Stroop task: PET activation studies. NeuroImage, 6, 81-92. doi:10.1006/nimg.1997.0285

[31] Mead, L.A., Mayer, A.R., Bobholz, J.A., Woodley, S.J., Cunningham, J.M., Hammeke, T.A., et al. (2002). Neural basis of the stroop interference task: Response competition or selective attention. Journal of International Neuropsychological Society, 8, 735-742. doi:10.1017/S1355617702860015 